\title{
Computational Analysis of Smile Weight Distribution across the Face for Accurate Distinction between Genuine and Posed Smiles
}

\author{
Ahmad Al-dahoud \\ Center of Visual Computing \\ University of Bradford \\ Bradford, BD7 1DP, UK \\ a.a.a.al-dahoud@bradford.ac.uk
}

\author{
Hassan Ugail \\ Center of Visual Computing \\ University of Bradford \\ Bradford, BD7 1DP, UK \\ h.ugail@bradford.ac.uk
}

\begin{abstract}
In this paper, we report the results of our recent research into the understanding of the exact distribution of a smile across the face, especially the distinction in the weight distribution of a smile between a genuine and a posed smile. To do this, we have developed a computational framework for the analysis of the dynamic motion of various parts of the face during a facial expression, in particular, for the smile expression. The heart of our dynamic smile analysis framework is the use of optical flow intensity variation across the face during a smile. This can be utilised to efficiently map the dynamic motion of individual regions of the face such as the mouth, cheeks and areas around the eyes. Thus, through our computational framework, we infer the exact distribution of weights of the smile across the face. Further, through the utilisation of two publicly available datasets, namely the CK+ dataset with 83 subjects expressing posed smiles and the MUG dataset with 35 subjects expressing genuine smiles, we show there is a far greater activity or weight distribution around the regions of the eyes in the case of a genuine smile.
\end{abstract}

Keywords - Smile Analysis; Genuine Smiles; Optical flow.

\section{INTRODUCTION}

Across humanity, smiling is universal and is considered to be a sign of happiness. And as the saying "laughter is the best medicine" suggests, smile bears important ramifications with beliefs such as it makes one more attractive, less stressful even in upsetting situations and employers tending to promote people who smile more. There exist scientific research too that appear to validate such beliefs or claims. In fact, many traits appear to be encoded in the smile of a person such as the ability to win a fight [1], whether a couple would divorce [2] and even such traits as gender appear to be encoded in the smile [3, 4, 29].

An important aspect to bear in mind is that the predictably of much of such traits depends on whether the smile is genuine or not. In addition to this, it is rather important to understand the characteristics of a smile and the perceived meaning one must attach to it [5]. Bernstein et al., [6], for example, show that individual preferences to work with others can depend on the level of the genuine smile they bear. In one of their experiments, they showed participants videos of individuals expressing
Duchenne (genuine) and non-Duchenne (posed or polite) smiles and asked them to evaluate each individual as a potential partner for a project on which they might collaborate with. The results show participants have a greater preference to work with individuals displaying genuine smiles.

\section{RELATED WORK}

Being able to accurately distinguish between Duchenne and nonDuchenne smiles is often imperative, though the task appears to be far from easy [7]. To date, much work to distinguish between genuine and posed smiles and their effects have been undertaken. Dimberg [8] makes use of electromyographic (EMG) inferred reactions to classify Duchenne and nonDuchenne smiles, identifying the muscles related to these smiles in the context of other emotions such as disgust, fear, anger, sadness and surprise. The results indicate that both types of smiles have a high occurrence in the surprise and pleasure expressions. However, it was noted that Duchenne smiles have significantly stronger EMG activity in the periocular and cheek muscle regions. Similarly, Mai et al., [9] investigated Chinese participants to judge for Duchenne and non-Duchenne by focusing on the mouth and the eyes. In their experiment, 100 participants were asked to evaluate 20 videos and were asked to rate each video regarding the genuineness of the smile being expressed. Afterwards, the participants were asked to answer the question, "what part of the face was most useful for discriminating between fake and real smiles?" Results indicate that participants acquire more information from the eyes to successfully distinguish between genuine and polite smiles.

From a computational standpoint, the above results, particularly that by Mai et al., [9], is probably unsurprising. There has been significant work in terms of utilising computer based facial analysis to understand facial emotions including the dynamics of smiles. In this sense, the use of the facial action coding system, (FACS) and the original work of Ekman [10] is particularly noteworthy. FACS, originally developed by Hjortsjö in 1969 [11], is a system that is used to categorise 


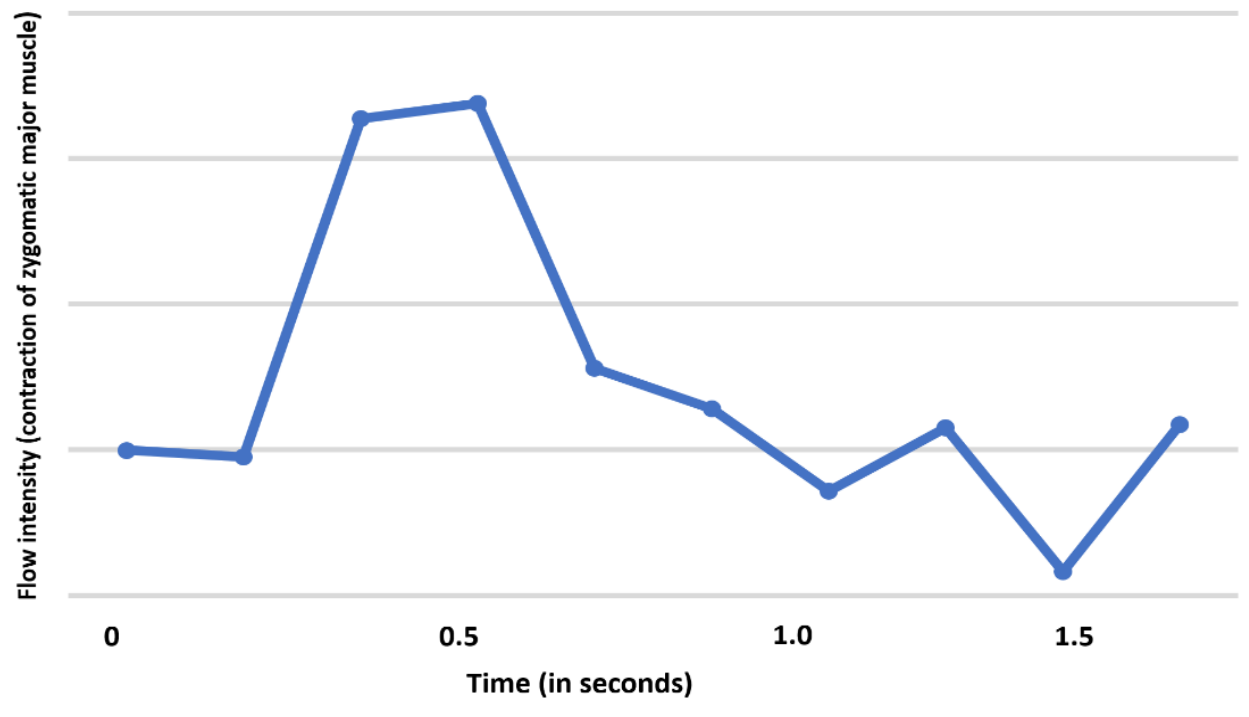

Figure 1: The dynamics of a typical smile computed using the optical flow intensity - onset to apex to offset.

human facial emotions based on the underlying facial muscular movement which was widely adopted by Ekman, e.g. Ekman and Friesen [12]. Accordingly, FACS represents the facial expressions through the muscular movement called "Action Units (AUs)". Facial expressions can be measured by observing these AUs, and according to Ekman's initials model, there are as many as 46 AUs, whose values - on their own or in combination - can accurately quantify facial emotions.

There is ample evidence to suggest that the genuine smiles associated with enjoyment produce increased tension in the zygomatic major and the orbicularis oculi muscles [13, 14] which regarding the action units is identified by the cheek raiser, also known as the Duchenne marker (AU6). The Duchenne marker signifies the appearance of crow's feet around the eyes as a result of closing and opening of the eyes giving rise to wrinkles around them. On the other hand, a non-Duchenne smile appears to be formulated by the upward movement of mouth corners (AU12). To this end, Wu et al., [15] have proposed a computational system to identify the genuineness of a smile based on the detection of AUs 6 and 12. Based on the 2D principal component analysis combined with Gabor filters and support vector based machine learning they report an $85.9 \%$ accuracy in smile classification. Similarly, Nakano et al., [16] utilized principle component analysis along with neural network based machine learning to achieve smile classification rates of up to $90 \%$, though their framework does not explicitly utilize FACS coding.

Inspired by the copious amount of work being undertaken in the area of automated facial emotion analysis in the field of visual computing, in this work, we set out to understand the dynamics of a human smile in great detail. In particular, the focus of our work is to understand how a smile is distributed across the face. By doing so, we hoped to verify the results of physical experiments for distinguishing between genuine and posed smiles. In addition to this, our aim is to create an efficient computational framework for accurately measuring the intensity or the weight distribution of a given smile.

This paper is structured as follows. In Section III we discuss in detail the methodology we have adopted to undertake a detailed analysis of facial expressions, in order to map the distribution of the smile across the face. More specifically, we discuss the dense optical flow based algorithm we have adopted for automated analysis of the dynamics of smile so as we can map the distribution of the smile across the face. In Section IV we discuss the experiments and the results of using our framework to analyse both genuine and posed smiles. Finally, in Section IV we provide a discussion and conclude the paper in Section V.

\section{OUR COMPUTATIONAL SMILE WEIGHT Distribution ANALYSIS FRAMEWORK}

During a smile, the zygomatic major muscle of the face contracts and the noticeable visual effect, as a result, is the raising of the lip corners. As far as the dynamics of a smile is concerned, there are three distinct phases in any type of a smile. They are, the onset (which starts from the neutral facial expression to the peak of zygomatic major muscle contraction), the apex (which refers to the time it takes for the smile to stay in the expressive state) and the offset (where the facial expression reaches from expressive state back to the neutral). Figure 1 illustrates the dynamics of a typical simile where the intensity of optical flow is utilised to demonstrate the variation of the smile. Further details of how optical flow is utilised discussed later in this section.

There are 18 distinct types of smiles and all of which must bear the three distinct phases mentioned above, though the effects pronounced on different parts of the face do differ in each case. For example, in a Duchenne (or a genuine) smile the cheeks get raised along with a significant narrowing of the eye aperture, 
resulting in wrinkles (or crow's feet) on the outer sides of the eyes [17]. analysis and output. The detection phase includes face detection, face resizing, landmark detection and identifying the

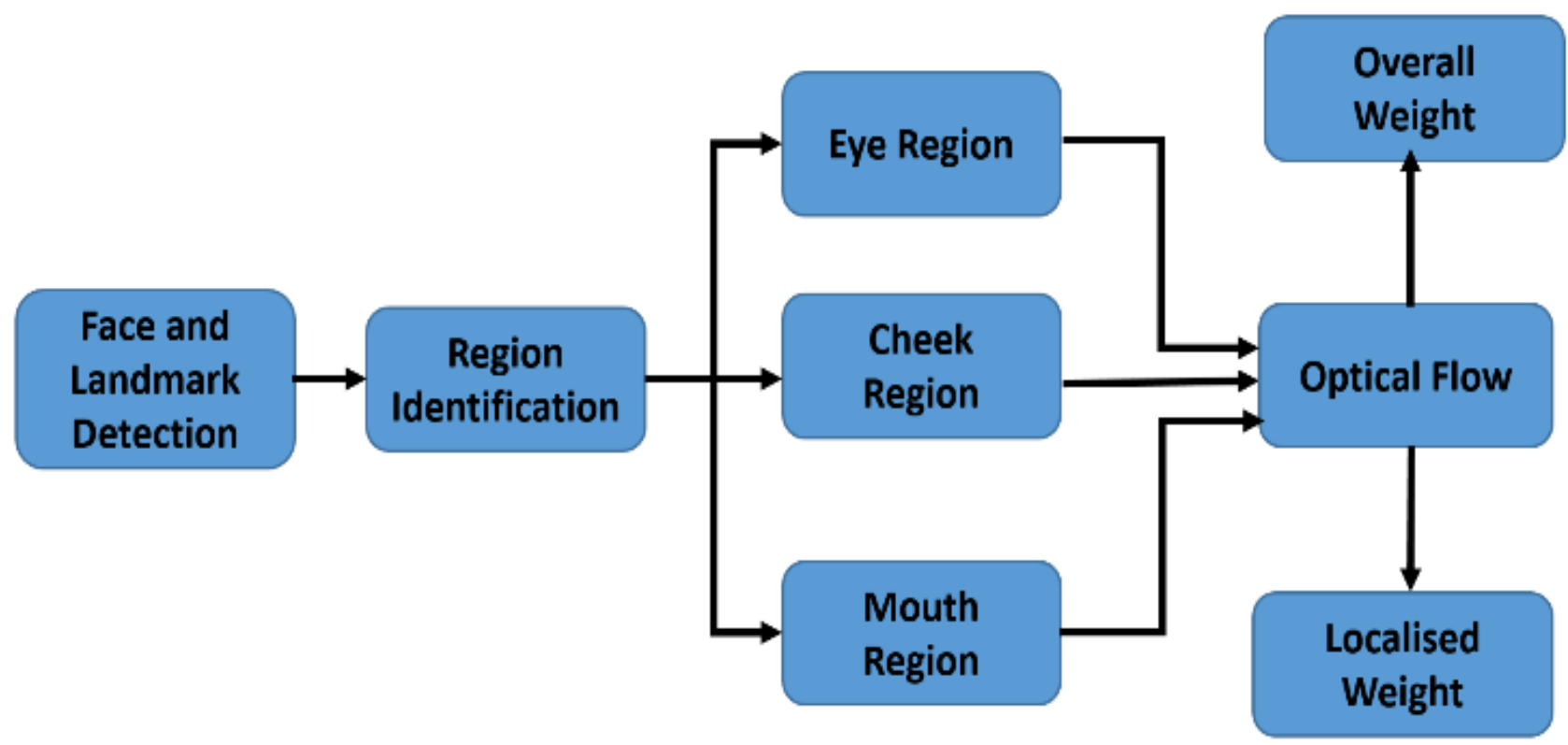

Figure 2: Description of our computational framework for analysis the smile dynamics.

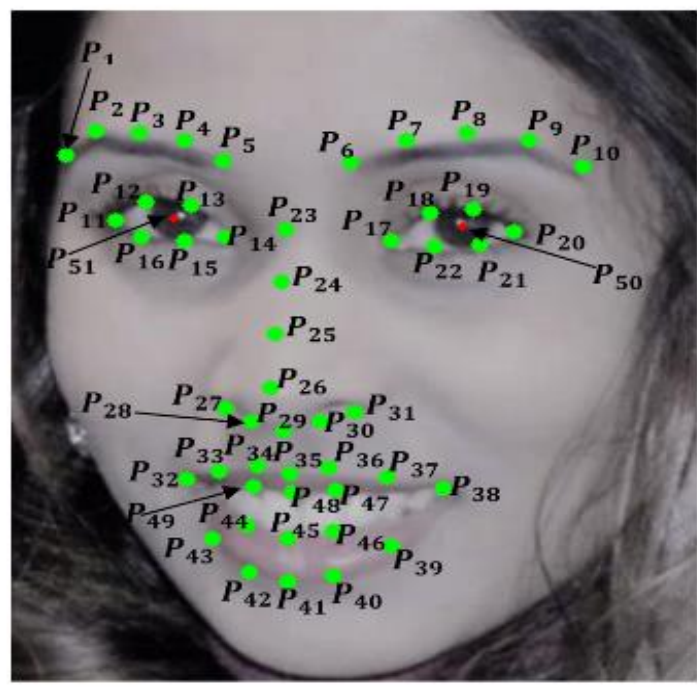

(a)

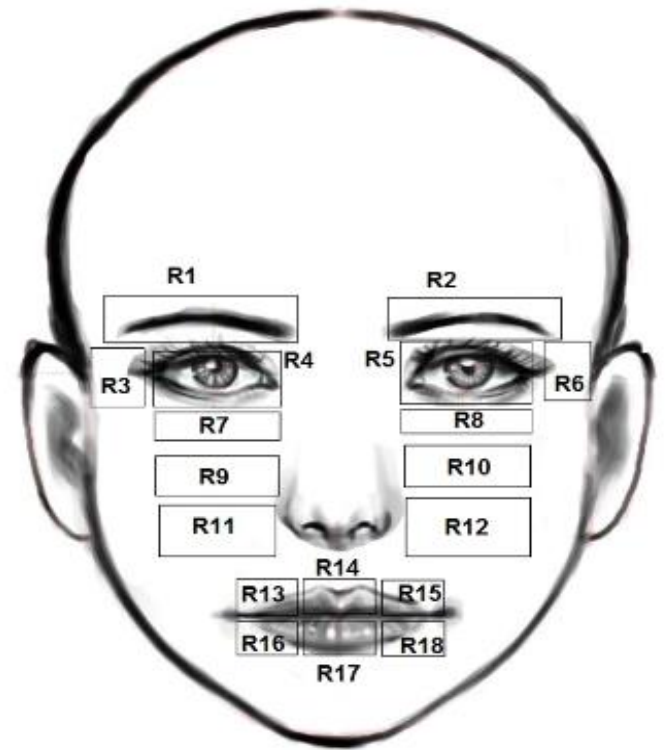

(b)

Figure 3: Description how landmarks are detected and how the relevant regions of interest on the face are determined.

The methodology we propose is to track the dynamic variations of the smile, especially the apex phase of the smile. Our framework is based upon computing the dynamic geometry changes across the faces, through the employment of a technique called optical flow analysis [18].

As shown in Figure 2, our computational analysis framework for smiles contains three main components namely, detection, regions of interest (ROI). The first step in our framework is to detect and track the face within a given video sequence. For this purpose, we have used a well known algorithm for image processing called the Viola-Jones algorithm [19]. It is based on Haar feature selection to create an integral image through the use of Adaboost training and cascade classifiers. The ability of this algorithm to robustly detect faces under different lighting conditions has been well established and our extensive 
experimentation using the algorithm have demonstrated to us that this algorithm is fairly robust to occlusions as well as variations in lighting conditions [20].

\begin{tabular}{|c|c|c|}
\hline Facial Features & ROI & Reference Landmarks \\
\hline \multirow{7}{*}{ Eyes } & R1 & $P_{1, X}, P_{2, Y}$ \\
\cline { 2 - 3 } & R2 & $P_{6, X}, P_{7, Y}$ \\
\cline { 2 - 3 } & R3 & $P_{12, X}, P_{1, Y}$ \\
\cline { 2 - 3 } & R4 & $P_{11, X}, P_{12, Y}$ \\
\cline { 2 - 3 } & R5 & $P_{17, X}, P_{18, Y}$ \\
\cline { 2 - 3 } & R6 & $P_{20, X}, P_{19, Y}$ \\
\hline Cheeks & R7 & $P_{11, X}, P_{16, Y}$ \\
\cline { 2 - 3 } & R8 & $P_{17, X}, P_{22, Y}$ \\
\cline { 2 - 3 } & R9 & $P_{32, X}, P_{32, Y}$ \\
\cline { 2 - 3 } & R10 & $P_{38, X}, P_{38, Y}$ \\
\cline { 2 - 3 } & R11 & $P_{32, X}, P_{32, Y}$ \\
\cline { 2 - 3 } & R12 & $P_{38, X}, P_{38, Y}$ \\
\hline Mouth & R13-R18 & $P_{32(X, Y)}, P_{33(X, Y)}, P_{35(X, Y)}$, \\
& & $P_{37(X, Y)}, P_{38(X, Y)}, P_{39(X, Y)}$, \\
\hline
\end{tabular}

Table 1: Description of the regions of interest, the relevant facial features and the corresponding facial landmars.

After detecting the face, we resize it to a window of 448 by 448 pixels. This is to ensure uniformity is maintained across all the ROI and that landmark detection is undertaken appropriately for each frame of video consisting a face that we must analyse. Landmark detection is done using the CHEHRA model [21], which is a machine learning algorithm used to detect the facial landmarks as shown in Figure 3(a).

The CHEHRA model algorithm has been trained to detect facial landmarks using "faces in-the-wild datasets" under varying illumination, facial expressions and head pose. Again, we undertook many tests to evaluate the performance of the algorithm. We found that, in general, the algorithm is acceptable though we noticed that it is unlikely to be fully applicable for real time face analysis applications.

Upon detection of the landmarks, we define the relevant ROI such as the area around the eyes, cheeks and the mouth. To do this, we first identify all the landmarks in a neutral facial expression which helps us to identify the initial location of the mouth, cheeks and eyes. This process also enables us to normalise the ROI identification process.

Table 1 shows the relevant facial features (i.e. the mouth, eyes and the cheeks) and the associated ROI as well as the corresponding landmarks. The computation of a specific ROI is done through two steps. First, we locate the relevant reference landmarks which are defined as two 2-dimensional vectors $(x, y)$ where variation across each axis is considered separately. Second, using the formulation $\left(\lambda_{x}, \lambda_{y}\right)$ we can denote a shift distance for each axis from the reference points.
Thus, using the shift distances $\left(\lambda_{x}, \lambda_{y}\right)$ along with the origin of the a central landmark point, we can compute the ROI using Equation (1) such that,

$R O I=\left\{\begin{array}{c}O_{x}=P_{x} \pm \lambda_{x} \\ R O_{y}=P_{y} \pm \lambda_{y}\end{array}\right.$

where $P_{x}$ shows the reference point along the x-axis and $P_{y}$ shows the reference point along the y-axis.

It should be noted that there are ROI where no obvious boundaries can be defined such as the R9, R10, R11, R12 and that below the eyes, R7 and R8. In such cases, where landmarks cannot be directly identified, we use the Euclidean wise nearest reference landmark. For example, to locate the left eyebrows, using landmark $P_{1}$ we allocate a window of appropriate size (110 by 35 pixels in this case) which covers the area surrounding the eyebrows. Similarly, to locate the right cheek, we use the mouth right corner point $P_{38}$ as the reference landmark to infer R12, R10 and R8.

Using the above formulation and by means of using the facial landmarks as reference points, we thus allocate the ROI as shown in Figure 3(b). Moreover, we identify 18 ROI through which the motion around mouth, cheeks and eyes can be monitored. In particular, we identify 4 different ROI around the eye, i.e. eyebrow (R1 and R2), eye corners (R4, R5) and (R3, R6) and the area beneath the eye (R7, R8). The reason we allocate such greater number of ROI to the eye area is that we wanted to study the regions around the eye area in greater detail. This is because previous work has shown that there are greater distinctions in the level of activity around eye area between genuine and non-genuine smiles. Therefore, to test this as well as to fine tune our framework, to distinguish between genuine and non-genuine smiles we wanted to study the area around the eyes in greater detail.

Once the relevant ROI are allocated its motion over time (through the dynamics of a smile) can be tracked and analysed. To do this, we apply the optical flow algorithm by Farnebäck [22] where the algorithm is applied to all ROI. The particular optical flow algorithm we use is a two-frame motion estimation algorithm. It uses quadratic polynomials to approximate the motion between two sequence of frames to approximate each neighbourhood pixel movement of both frames. Furthermore, Farnebäck's algorithm is considered to be a dense optical flow algorithm since it computes the optical flow for all pixels in the image which is considered to be more accurate for motion data analysis when compared to for example, to sparse algorithms like Lucas Canade algorithm [23]. Using Farnebäck's algorithm, we can estimate the successive motion for two attributes, i.e. displacement values and movement occurrences for each facial feature.

As highlighted above, Farnebäck's approach to optical flow computation encompasses all pixels available in an image to identify the relevant motion. The approach adopted is to 
approximate the neighbourhood using quadratic polynomial expansions. The following equation shows how the motion is expressed as a local "signal" model and within a local coordinate system such that,

$f(x)=x^{T} A x+b^{T} x+c$,

where $A$ is a symmetric matrix, $b$ is a vector and $c$ is a scalar. The coefficients are estimated by weighted least square fit to single pixel values around a given neighbourhood. Based on the hypothesis, if a polynomial undergoes an ideal translation taking into consideration the exact quadratic polynomial form such that,

$$
f_{1}(x)=x^{T} A_{1} x+b_{1}^{T} x+c_{1}
$$

a new signal $f_{2}$ can be constructed by a global displacement $d$, such that,

$$
f_{2}(x)=x^{T} A_{2} x+b_{2}^{T} x+c_{2}
$$

To estimate the displacement value $d$, the neighbourhood pixels of the point $(x, y)$ and $(x+d x, y+d x)$ can be considered.

Once the optical flow values are computed, they need to be normalised in order to overcome some of the challenging factors such as the face location relative to the camera, the changing size of the face as it moves from one frame to the other. To obtain a uniform normalisation, we use one of the most stable areas of the face namely, the triangular area formed with the tip of the nose, $P_{29}$ and the two eye corners, $P_{11}$ and $P_{20}$.

\section{EXPERIMENTS AND RESULTS}

To test the validly of our facial feature tracking approach for smile weight distribution analysis, we used two sets of data which are commonly utilised for emotion analysis research. They are the CK+ [24] and MUG dataset [25].

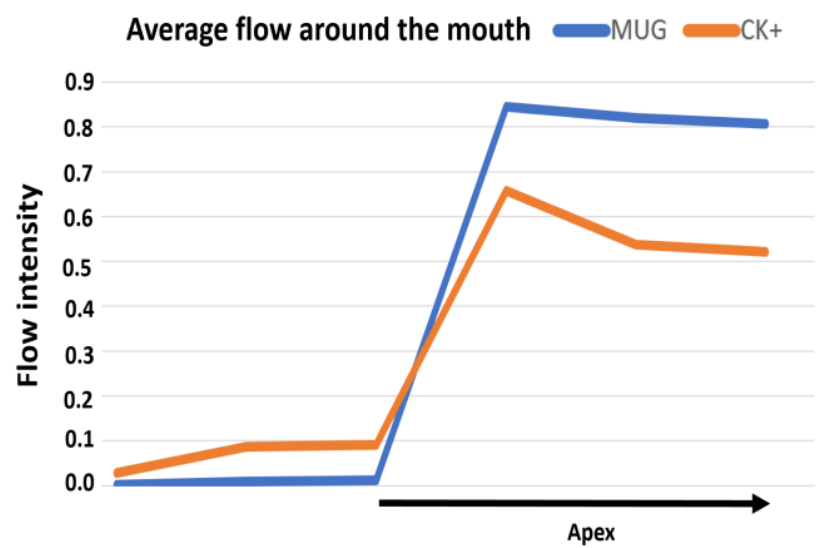

Figure 4: Average flow around the mouth area for the subjects in the MUG and the $\mathrm{CK}+$ datasets.
The CK+ dataset has a total of 54 subjects, consisting of 27 females and 27 males with posed facial expressions. Each subject went from the neutral expression to the peak of the expression. In our experiments, we have used the posed (nongenuine) smile data from the $\mathrm{CK}+$ dataset in order to analyse posed smiles.

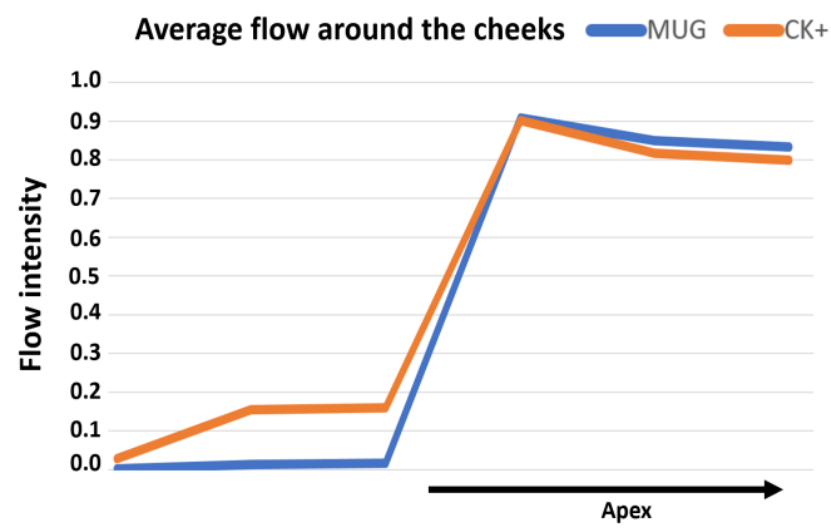

Figure 5: Average flow around the cheeks for the subjects in the MUG and the CK+ datasets.

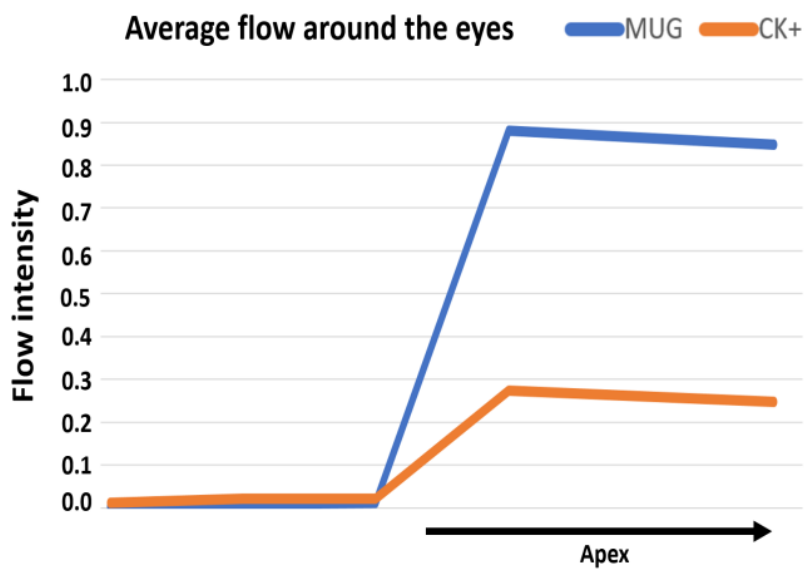

Figure 6: Average flow around the eyes for the subjects in the MUG and the $\mathrm{CK}+$ datasets.

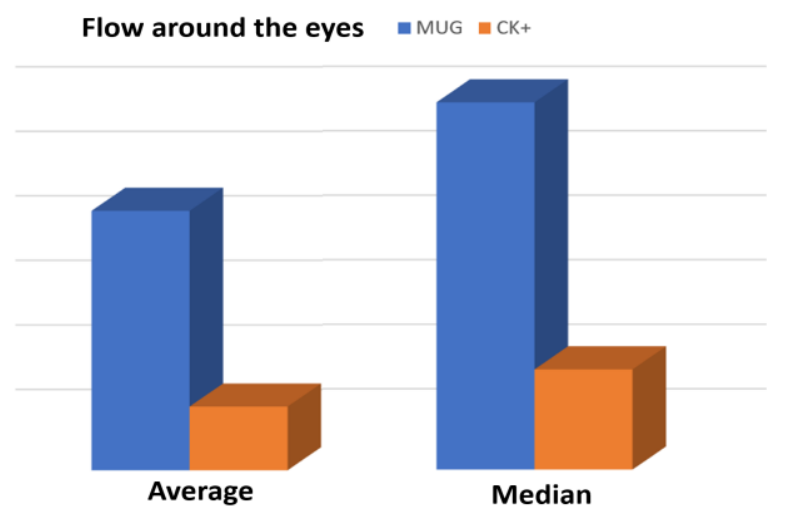

Figure 7: Average and median flow around the eyes for the subjects in the MUG and the $\mathrm{CK}+$ datasets. 


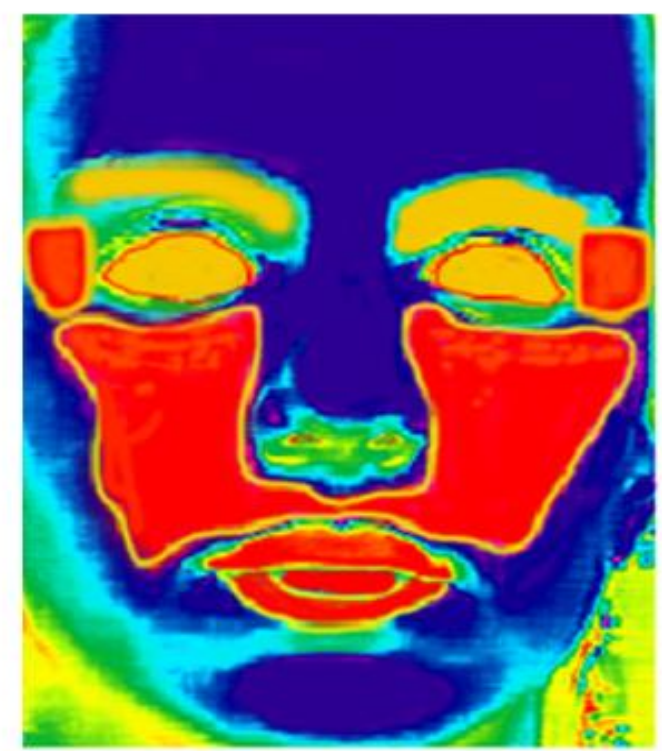

Non-Duchenne Smile
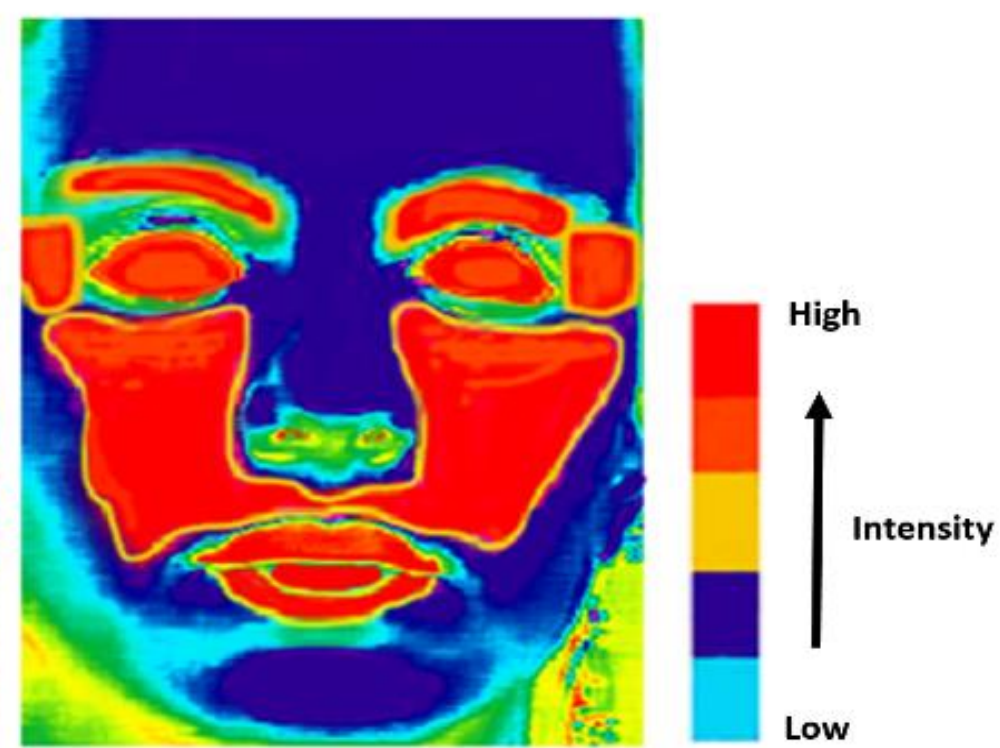

\section{Duchenne Smile}

Figure 8: Heat map based weight intensity distribution across the face for genuine and posed smile.

On the other hand, the MUG dataset contains a total of 26 subjects, consisting of 13 females and 13 males. Each subject went from the natural expression and returned to it, and in all cases, the smiles were non-posed, where the subjects were recorded while they were watching a video that was created in order to induce emotions.

To analyse the weight distribution of the smile, we particularly focused on the apex portion of the smile where the zygomatic major muscle is most stretched. We computed the flow around the regions of the mouth, eyes, as well as the cheeks for each subject in each of the datasets. Then, we computed the averages for each dataset to see if there are distinct differences between flow intensities, not only in the various regions of the face but also for comparison between genuine and posed smiles.

Figure 4 shows the intensity of the optical flow motion around the mouth area averaged over all the subjects in the datasets. As it can be observed, when the apex of the smile is considered, there are some differences in the mouth area between a genuine and a posed smile in that there is a greater overall activity for a genuine smile.

Figure 5 shows the intensity of the optical flow motion around the cheeks area averaged over all the subjects in the datasets. As it can be observed, when the apex of the smile is considered, there are no distinct differences in the flow intensities around cheeks between a genuine and a posed smile. Hence we can conclude that the flow around the cheeks is probably not appropriate to consider to distinguish between genuine and posed smiles.

Similarly, in Figure 6, we show the intensity of the optical flow distribution for both the datasets where we have averaged the flow distribution. As can be observed from the results shown in Figure 6, there is a significant difference in the intensity of the flow in the eye areas between genuine and posed smiles.

In Figure 7, we show the bar graphs of the flow intensity variation around the eye area. Here we have computed both the averages as well as the median for both the datasets. Again as shown in Figure 6, the intensity of flow around the eyes is significantly higher for genuine smiles.

Finally, in Figure 8, we depict a heat map for a typical smile for both genuine and posed smile, in order to indicate the intensity of the flow distribution across various regions of the face, for genuine and posed smiles.

\section{DISCUSSIONS}

In recent times, attention to the detailed analysis of facial expressions has become a topic of great interest in the area of visual computing. Since smile is the most prominent facial expression within social contexts, the study of smile facial expression has received far greater attention. In this sense, understanding the weight distribution of a smile across the face along with an understanding of the distinction between genuine and posed smiles is a topic of great interest.

Past as well as the more recent work in this area suggest that researchers have looked at the symmetry, timing and speed of smiles in order to understand the dynamics of smiles in detail [26, 27, 28]. Such work is undertaken using generic feature detection and processing and sometimes by the employment of machine learning techniques. 
However, in this work, we show that the application of dense optical flow on ROIs across the face can provide us with an efficient mechanism to analyse the weight distribution of a smile. Our experiments do confirm that the vast majority of the activity, in a genuine smile, does occur around the eye areas. This is indeed verifiable by the relevant physical experiments such as the use of EMG. Hence, we show our framework, though lightweight, can be utilised for a deeper understanding of facial emotions, especially the weight distribution of smiles via facial dynamics.

\section{CONCLUSIONS}

In this paper, we discuss a computational framework to analyse facial expressions, in particular, the smile expressions. More specifically, we show how the weight distribution of the smile across the face can be mapped for a more detailed understanding of the activities of various facial regions. The heart of our computational framework is the deployment of a dense optical flow algorithm which is applied across specific regions of the face which are automatically identified through facial landmark detection.

One major aim of this work is to computationally understand as well as verify the distinction between genuine and posed smiles. From our experiments, through the use of two datasets, namely the $\mathrm{CK}+$ and the MUG dataset, we infer that, during a genuine smile, there is significant facial dynamic activity around the eye area of the face. It is noteworthy that this is in line with the physical experiments which confirm that during genuine smiles there is a significant closure of eyes and significant tension in the part of the zygomatic major muscle around the eye area.

This work can be taken further forward in various directions. One direction we feel this work should be taken forward is the detailed analysis of the smile dynamics through extensive experimentation using the framework we have described. For example, we have just studied the flow intensity during the apex phase of the smile. It would be interesting to look at the onset as well as the offset phases of the smile too. Further, it will be interesting to use more sophisticated analysis techniques, beyond simple averaging and median computations, for a deeper understanding of the dynamics of smiles and their detailed weight distributions.

\section{ACKNOWLEDGEMENTS}

This work was supported in part by the European Union's Horizon 2020 Programme H2020-MSCA-RISE-2017, under the project PDE-GIR with grant number 778035.

\section{REFERENCES}

1. M.W. Kraus and T-W. D. Chen, "A winning smile? Smile intensity, physical dominance, and fighter performance," Emotion, 13(2), 270-279. 2013.

2. M. J. Hertenstein, C. A. Hansel, A. M. Butts and S. N. Hile, "Smile intensity in photographs predicts divorce later in life," Motivation and Emotion, 33(2), 99-105, 2009.

3. H. Ugail and A. Al-dahoud, "Is gender encoded in the smile? A computational framework for the analysis of the smile driven dynamic face for gender recognition," The Visual Computer, https://doi.org/10.1007/s00371-018-1494-x, 2018.

4. A. Al-dahoud and H. Ugail, "On gender identification using the smile dynamics," Proceedings of Cyberworlds 2017 Conference, IEEE, 2017.

5. B. Duchenne, "The mechanism of human facial expression," Cambridge, U.K, Cambridge University Press, 1990.

6. M. J. Bernstein, D. F. Sacco, C. M. Brown, S. G. Young and H. M. Claypool, "A preference for genuine smiles following social exclusion," Journal of Experimental Social Psychology, 46(1), 196-199, 2010.

7. R. Soussignan and B. Schaal, "Forms and social signal value of smiles associated with pleasant and unpleasant sensory experience," Ethology, 102(8), 1020-1041, 1996.

8. U. Dimberg, "Facial electromyographic reactions and autonomic activity to auditory stimuli," Biological Psychology, 31(2), 137-147, 1990

9. X. Mai, Y. Ge, L. Tao, H. Tang, C. Liu and Y-J. Luo, "Eyes are windows to the Chinese soul: Evidence from the detection of real and fake smiles," PloS one, 6(5), e19903, 2011.

10. P. Ekman and W.V. Friesen, "The facial action coding system: a technique for the measurement of facial movement," San Francisco, CA, USA: Consulting Psychology Press Inc., 1978.

11. C-H. Hjortsjö, "Man's face and mimic language," Studen litteratur, 1969.

12. P. Ekman, R.J. Davidson, and W.V. Friesen, "The Duchenne smile: Emotional expression and brain physiology II," Journal of Personality Social Psychology, 58(2), 342-353, 1990.

13. V. Surakka and J.K. Hietanen, "Facial and emotional reactions to Duchenne and non-Duchenne smiles," International Journal of Psychophysiology, 29(1), 23-33, 1998. 
14. U. Dimberg, M. Thunberg and K. Elmehed, "Unconscious facial reactions to emotional facial expressions," Psychological Science, 11(1), 86-89, 2000.

15. P. Wu, W. Wang and H. Liu, "Methods of recognizing true and fake smiles by using AU6 and AU12 in a holistic way," in Proceedings of 2013 Chinese Intelligent Automation Conference: 2013, Springer, 603-613, 2013.

16. M. Nakano, Y. Mitsukura, M. Fukumi and N. Akamatsu, "True smile recognition system using neural networks," in Neural Information Processing, 2002 ICONIP'02 Proceedings of the 9th International Conference, IEEE, 650-654, 2002.

17. Z. Ambadar, J. F. Cohn, and L. I. Reed, "All smiles are not created equal: Morphology and timing of smiles perceived as amused, polite, and embarrassed/nervous," Journal of Nonverbal Behavior, 33(1), 17-34, 2009.

18. D. Fortun, P. Bouthemy and C. Kervrann, "Optical flow modeling and computation: A survey," Computer Vision and Image Understanding, 134, 1-21, 2015.

19. P. Viola and M. J. Jones, "Robust real-time face detection," International Journal of Computer Vision, 57(2), 137-154, 2004.

20. A. Al-dahoud and H Ugail, "A Method for Location Based Search for Enhancing Facial Feature Detection," In Advances in Computational Intelligence Systems, Springer, pp. 421-432, 2016.

21. A. Asthana, S. Zafeiriou, S. Cheng and M. Pantic, "Incremental face alignment in the wild", In CVPR 2014 https://ibug.doc.ic.ac.uk/resources/chehra-tracker-cvpr-2014/ , 2014.

22. G. Farnebäck, "Two-frame motion estimation based on polynomial expansion,” Image Analysis, 363-370, 2003.

23. B. D. Lucas and T. Kanade, "An iterative image registration technique with an application to stereo vision," In: IJCAI: 1981, 674-679, 1981.

24. P. Lucey, J. F. Cohn, T. Kanade, J. Saragih, Z. Ambadar and I. Matthews, "The extended Cohn-Kanade dataset $(\mathrm{CK}+)$ : A complete dataset for action unit and emotion-specified expression," In: Computer Vision and Pattern Recognition Workshops (CVPRW), IEEE, 2010, 94-101, 2010.

25. N. Aifanti, C. Papachristou and A. Delopoulos, "The MUG facial expression database," in Image Analysis for Multimedia Interactive Services (WIAMIS), 2010, IEEE, 1-4, 2010.

26. P. Ekman, J. C. Hager and W. V. Friesen, "The symmetry of emotional and deliberate facial actions," Psychophysiology, 18, 101-106, 1981.
27. K.L. Schmidt, S. Bhattacharya and R. Denlinger, "Comparison of deliberate and spontaneous facial movement in smiles and eyebrow raises," Journal of Nonverbal Behavior, 33, 35-45, 2009.

28. J.C. Borod, E. Koff and B. White, "Facial asymmetry in posed and spontaneous expressions of emotion," Brain Cognition, 2(2), 165-175, 1983.

29. H. Ugail, Secrets of a smile? Your gender and perhaps your biometric identity, Biometric Technology Today, 2018(6), 5-7, 2018. 